CORRIGENDUM

doi:10.1038/nature11667

\title{
Corrigendum: Reconstructing Native American population history
}

David Reich, Nick Patterson, Desmond Campbell, Arti Tandon, Stéphane Mazieres, Nicolas Ray, Maria V. Parra, Winston Rojas, Constanza Duque, Natalia Mesa, Luis F. García, Omar Triana, Silvia Blair, Amanda Maestre, Juan C. Dib, Claudio M. Bravi, Graciela Bailliet, Daniel Corach, Tábita Hünemeier, Maria Cátira Bortolini, Francisco M. Salzano, María Luiza Petzl-Erler, Victor Acuña-Alonzo, Carlos Aguilar-Salinas, Samuel Canizales-Quinteros, Teresa Tusié-Luna, Laura Riba, Maricela Rodríguez-Cruz, Mardia Lopez-Alarcón, Ramón Coral-Vazquez, Thelma Canto-Cetina, Irma Silva-Zolezzi, Juan Carlos Fernandez-Lopez, Alejandra V. Contreras, Gerardo Jimenez-Sanchez, Maria José Gómez-Vázquez, Julio Molina, Ángel Carracedo, Antonio Salas, Carla Gallo, Giovanni Poletti, David B.Witonsky, Gorka Alkorta-Aranburu, Rem I. Sukernik, Ludmila Osipova, Sardana A. Fedorova, René Vasquez, Mercedes Villena, Claudia Moreau, Ramiro Barrantes, David Pauls, Laurent Excoffier, Gabriel Bedoya, Francisco Rothhammer, Jean-Michel Dugoujon, Georges Larrouy, William Klitz, Damian Labuda, Judith Kidd, Kenneth Kidd, Anna Di Rienzo, Nelson B. Freimer, Alkes L. Price \& Andrés Ruiz-Linares

Nature 488, 370-374 (2012); doi:10.1038/nature11258

At the time of publication of this Letter, the authors were unaware of a manuscript arriving at broadly similar conclusions based on allotype analysis by Williams et al. ${ }^{1}$, which appeared in the American Journal of Physical Anthropology.

1. Williams, R. C. et al. GM allotypes in Native Americans: evidence for three distinct migrations across the Bering land bridge. Am. J. Phys. Anthropol. 66, 1-19 (1985). 